\title{
Expression of surfactant protein-A in exhaled breath condensate of patients with chronic obstructive pulmonary disease
}

\author{
XUE-FENG LIN ${ }^{1 *}$, LEI ZHANG ${ }^{2 *}$, SHU-YUAN SHI ${ }^{2 *}$, YI-CHU FAN $^{2}$, \\ ZHEN-LIN WU ${ }^{2}$, XUN ZHANG ${ }^{3}$ and DA-QIANG SUN ${ }^{3}$ \\ ${ }^{1}$ Department of Nursing, Tianjin Medical College, Tianjin 300222; ${ }^{2}$ Graduate School of Tianjin Medical University, \\ Tianjin 300070; ${ }^{3}$ Department of Thoracic Surgery, Tianjin Chest Hospital, Tianjin 300222, P.R. China
}

Received December 5, 2014; Accepted November 2, 2015

DOI: $10.3892 / \mathrm{mmr} .2015 .4702$

\begin{abstract}
Pulmonary surfactant protein A (SP-A) has been associated with host defense in the lung, and contributes to the pathogenesis of chronic obstructive pulmonary disease (COPD). The present study aimed to determine a non-invasive method of measurement of SP-A, and further examine the expression levels of SP-A in patients with COPD. SP-A was detected in the exhaled breath condensate (EBC) obtained from patients with COPD and from non-COPD subjects. The individuals recruited for the present study comprised 60 subjects with and without COPD, who underwent lobectomy for a solitary peripheral lung nodule. EBC was collected using a condenser, and an enzyme-linked immunosorbent assay (ELISA) was used to measure the levels of SP-A. Tissue samples were obtained during lobectomy through resection of the adjacent lung tissues, located $>5 \mathrm{~cm}$ from the nodule. Western blot analysis and immunohistochemistry were used to measure SP-A and SP-A-positive type II pneumocytes. The results demonstrated that SP-A was detectable in the EBC of all subjects. The results of the ELISA and western blotting demonstrated that the expression levels of SP-A were significantly decreased in patients with COPD, compared with the non-COPD subjects. The reduction of SP-A-positive type II pneumocytes was associated with the expression levels of SP-A. Decreased expression levels of SP-A in EBC were associated with a higher degree of airway limitation. These results suggested that the measurement of SP-A levels in the EBC may serve as a method for monitoring airway obstruction in patients with COPD. Further investigations are required in
\end{abstract}

Correspondence to: Professor Da-Qiang Sun, Department of Thoracic Surgery, Tianjin Chest Hospital, 261 Taierzhuangnan Road, Jinnan, Tianjin 300222, P.R. China

E-mail: sundaqiang999@163.com

${ }^{*}$ Contributed equally

Key words: chronic obstructive pulmonary disease, exhaled breath condensate, surfactant protein-A, type II pneumocytes order to examine these observations further and to elucidate the underlying mechanisms.

\section{Introduction}

Chronic obstructive pulmonary disease (COPD) is one of the leading causes of morbidity and mortality worldwide, characterized by poorly reversible airflow limitation $(1,2)$. The pathophysiology of COPD remains to be fully elucidated, which limits the development of a more effective therapies and novel drugs for COPD. Various methods have been used for diagnosing, monitoring and evaluating COPD, predominantly comprising indirect methods, including lung function tests (3) and imaging techniques (high resolution computed tomography) (4), and more direct evaluations using invasive measurements (bronchoscopy and induced sputum) $(5,6)$. Indirect and direct evaluation methods have adverse effects through exposure to noxious agents and the invasiveness of the sampling procedures, respectively (7). Therefore, the identification of biomarkers for COPD has received increasing attention, aiming to reflect the degree of disease severity with non-invasive measurements, and to assist in monitoring pharmacological therapy (8). Exhaled air and blood analysis may be used to develop biomarkers of lung diseases.

Exhaled breath condensate (EBC), obtained through cooling exhaled air during spontaneous breathing, offers promising real-time measurement of pulmonary pathobiology. The advantages of EBC include easy and non-invasive collection from patients using a portable device, and its suitability for sequential and longitudinal sampling of the lower respiratory tract $(9,10)$.

Pulmonary surfactant proteins, defined as SP-A, SP-B, SP-C and SP-D, are synthesized by type II pneumocytes, and SP-A and SP-D have been associated with the host defense of the lung (11-13). SP-A and SP-D lectins have been reported to contribute to the pathogenesis of COPD $(14,15)$, and abnormal inflammatory responses and the involvement of inflammatory cells and cytokines may affect the composition and function of surfactant proteins (16). The present study focused on SP-A, which functions in protecting the lungs from oxidants and inflammatory and infectious stress $(17,18)$. The immunomodulatory protein, SP-A, in bronchoalveolar lavage (BAL) fluid has also been reported to be associated with impaired 
functions in chronic smokers $(19,20)$. The preoperative detection methods of SP-A are limited, and predominantly include blood analysis and BAL fluid analysis (18-21). However, blood analysis only indirectly reflects the state of the lung, and BAL fluid analysis cannot be used as a routine procedure due to the invasiveness of the sampling procedure (22). Therefore, a novel non-invasive method, which is easy to perform, is required to measure the expression levels of SP-A and to routinely monitor the severity of COPD.

The present study aimed to measure levels of SP-A in the EBC of patients with COPD, to evaluate whether the expression levels of SP-A in EBC are associated with those in the lung tissue, and to assess whether SP-A in EBC is associated with lung function.

\section{Materials and methods}

Subjects. The population recruited for the present study comprised 60 subjects who underwent lobectomy for a solitary peripheral lung nodule between September 2012 to January 2013. All patients were recruited from the Tianjin Chest Hospital (Tianjin, China). Patients who had lung infection or acute COPD exacerbation within the month prior to commencement of the present study were excluded. Patients suffering from severe diseases of the heart, brain, liver or kidney were also excluded.

The diagnosis of patients with COPD was performed, based on the following clinical symptoms: History of exposure to risk factors and laboratory examination. The severity of COPD was assessed according to the American Thoracic Society and European Respiratory Society Consensus Statement (23), where post-bronchodilator forced expiratory volume (FEV) in $1 \mathrm{sec} /$ forced vital capacity $\leq 70 \%$, and an FEV of $1-80 \%$ confirmed the presence of airflow limitation that was not fully reversible. The 60 subjects were divided into two groups, according to pirometric evaluation: A COPD group (28 cases) and a non-COPD group (32 cases) (Table I). Written informed consent was obtained from all subjects, and the protocol was approved by the Medical Ethics Committee of Tianjin Chest Hospital.

EBC. EBC ( $2 \mathrm{ml}$ ) was collected using a condenser (Respiratory Research, Inc., Austin, TX, USA), as previously described (24). Patients were asked to rinse their mouth thoroughly, and were asked not to eat food or take medicine containing nitrite or nitrate. This technique allows the non-invasive collection of the on-gaseous components of the expiratory air.

Tissue samples. Tissue samples of $\sim 1 \mathrm{~cm}^{3}$ were obtained during lobectomy at the Tianjin Chest Hospital (Tianjin, China) through resection of adjacent lung tissues located $>5 \mathrm{~cm}$ from the nodule. The tissue samples destined for western blotting were immediately snap-frozen in liquid nitrogen and stored at $-80^{\circ} \mathrm{C}$. The tissues samples for immunohistochemistry were fixed using $10 \%$ formalin buffer for $<24 \mathrm{~h}$. Then, each tissue block was embedded in paraffin. Sections of $5 \mathrm{~cm}$ were cut from the tissue samples, and then tissue sections underwent routine immunohistochemistry procedures.

Enzyme-linked immunosorbent assay (ELISA). ELISA was used to measure the expression levels of SP-A in the EBC
Table I. Patient characteristics.

\begin{tabular}{lcc}
\hline Characteristic & COPD & Non-COPD \\
\hline Total cases (n) & 28 & 32 \\
Male (n) & 15 & 18 \\
Age (mean, years) & $58.71 \pm 11.22$ & $61.81 \pm 8.33$
\end{tabular}

COPD, chronic obstructive pulmonary disease.

of all subjects, using a Surfactant Associated Protein A kit (Cloud-Clone Corp, Houston, TX, USA). Briefly, dilutions (100 $\mu \mathrm{l}$ each) of standard, blank or EBC samples were added to the appropriate well and incubated for $2 \mathrm{~h}$ at $37^{\circ} \mathrm{C}$. Subsequently, liquid from each well was removed, detection reagent $\mathrm{A}(100 \mu \mathrm{l})$ was added to the wells of a micro-titer plate and incubated at $37^{\circ} \mathrm{C}$ in the dark for $60 \mathrm{~min}$. Following washing with $350 \mu \mathrm{l}$ of $1 \mathrm{X}$ wash solution, detection reagent $\mathrm{B}$ (100 $\mu \mathrm{l})$ was added to the wells, and the plate was incubated for a further $30 \mathrm{~min}$ at $37^{\circ} \mathrm{C}$ in the dark. Subsequently, $90 \mu \mathrm{l}$ substrate solution was added to the wells and incubated for $15-25 \mathrm{~min}$ at $37^{\circ} \mathrm{C}$ in the dark. The reaction was terminated following the addition of $50 \mu \mathrm{l}$ stop solution, and the absorbance was measured at $450 \mathrm{~nm}$ using a microplate reader 550 (Bio-Rad Laboratories, Inc., Hercules, CA, USA).

Western blot analysis. The expression levels of SP-A were detected using western blot analysis, following standard procedures. Briefly, fresh lung tissues were homogenized in radioimmunoprecipitation assay buffer (Beyotime Institute of Biotechnology, Shanghai, China) and protein concentrations were determined using the Bradford assay (Bio-Rad Laboratories, Inc.). Equal quantities of protein $(40 \mu \mathrm{g})$ were separated by $12 \%$ SDS-PAGE (SDS-PAGE Gel Preparation kit; Beyotime Institute of Biotechnology) and transferred to nitrocellulose blotting membranes (Schleicher \& Schuell Bioscience GmbH, Dassel, German). The membranes were then blocked by incubating with $10 \%$ nonfat dried milk overnight at $4^{\circ} \mathrm{C}$. Subsequently, the membranes were incubated with mouse anti-human SP-A monoclonal antibody (1:500; cat. no. PE-10; Fuzhou Maixin Biotech Co., Ltd.) or mouse anti-actin antibody (1:1,000; cat. no. MAB1501; EMD Millipore, Billerica, MA, USA) at room temperature for $1 \mathrm{~h}$, followed by incubation with horseradish peroxidase-conjugated rabbit anti-mouse secondary antibody (1:1,000; cat. no. sc-358914; Santa Cruz Biotechnology, Inc., Dallas, TX, USA). All experiments were repeated in triplicate. The protein expression levels were quantified using Photoshop Image Analysis software (Image-Pro Plus 6.0; Media Cybernetics Inc., Rockville, MD, USA).

Immunohistochemistry. For immunohistochemical analysis, sections were deparaffinized and rehydrated, and antigen retrieval was achieved by boiling $\left(92-98^{\circ} \mathrm{C}\right)$ in citric acid buffer (pH 6.0) for $20 \mathrm{~min}$. Immunostaining of SP-A and thyroid transcription factor-1 (TTF-1) were performed using mouse anti-human SP-A monoclonal antibody (1:100; cat. no. PE-10; Fuzhou Maixin Biotech Co., Ltd.) and anti-TTF-1 monoclonal 


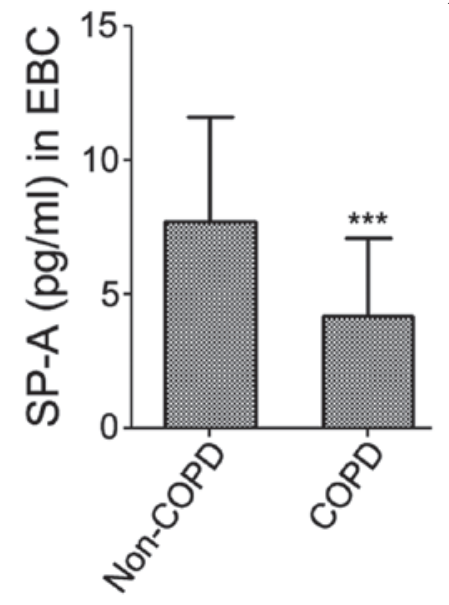

B

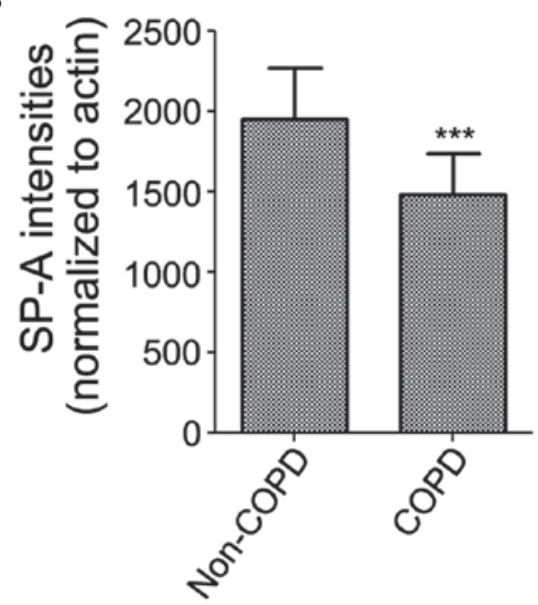

Figure 1. Expression levels of SP-A in EBC and lung tissue samples. (A) Expression levels of SP-A in the EBC of all subjects were collected and detected using an enzyme-linked immunosorbent assay. (B) Expression levels of SP-A in the lung tissues were measured using western blot analysis. Data are expressed as the mean \pm standard deviation. ${ }^{* * *} \mathrm{P}<0.001$. COPD, chronic obstructive pulmonary disease; EBC, exhaled breath condensate; SP-A, surfactant protein A.

A

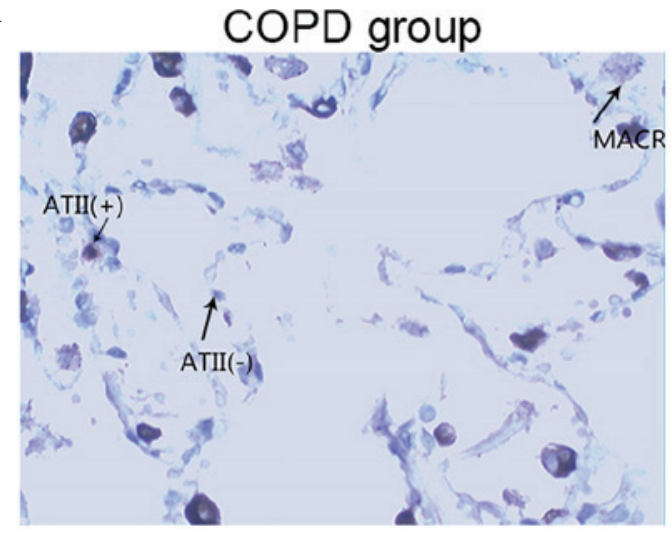

B

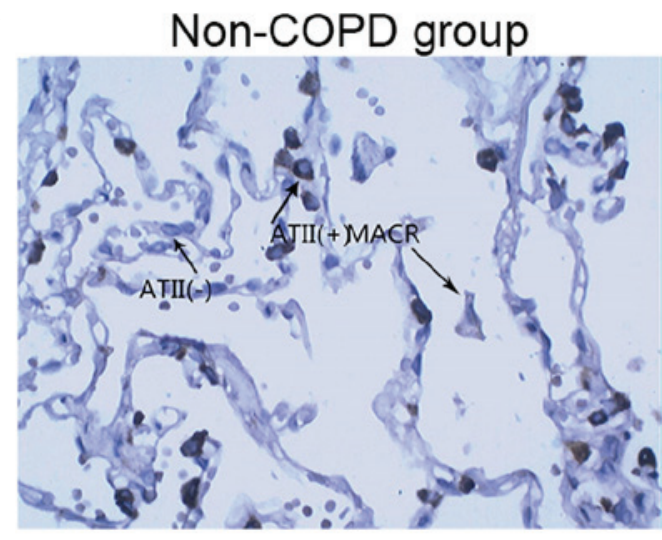

Figure 2. Immunostaining of SP-A in human lung tissue samples (magnification, $\mathrm{x} 400$ ). (A) SP-A-immunostained images from a patient with COPD and (B) a subject without COPD. ATII (+) indicates SP-A-positive type II pneumocytes; ATII (-) indicates SP-A-negative type II pneumocytes; MACR represents alveolar macrophages. COPD, chronic obstructive pulmonary disease; SP-A, surfactant protein A.

antibody (1:100; cat. no. MX011; Fuzhou Maixin Biotech Co., Ltd.), according to manufacturer's instructions. The sections were counterstained with Mayer's hematoxylin (Fuzhou Maixin Biotech Co., Ltd.). TTF-1 was used to count the total type II pneumocytes including the SP-A-positive type II cells on the adjacent serial sections.

Evaluation of the SP-A-positive type II pneumocytes was performed using a light microscope (BX50; Olympus Corporation, Tokyo, Japan) at x400 magnification (manual counting). A total of 20 microscopic fields on each slide were evaluated for all subjects, with the results expressed in cells/ $\mathrm{ml}^{2}$.

Statistical analysis. The results are expressed as the mean \pm standard deviation. Differences between the groups of subjects were determined using Student's t-test. Pearson's correlation coefficients were used to evaluate the associations between variables. The statistical software package, SPSS v.17 (SPSS, Inc. Chicago, IL, USA), was used for analysis. $\mathrm{P}<0.05$ was considered to indicate a statistically significant difference.

\section{Results}

SP-A in EBC and lung tissue samples. SP-A was detected in the EBC of all subjects. The results of the ELISA revealed that the expression levels of SP-A in the EBC were significantly decreased in the patients with COPD $(4.165 \pm 2.950 \mathrm{pg} / \mathrm{ml})$, compared with the non-COPD subjects $(7.706 \pm 3.923 \mathrm{pg} / \mathrm{ml}$; Fig. 1A). The protein expression levels of SP-A were subsequently examined in the lung tissue samples using western blot analysis. The protein expression levels of SP-A were significantly decreased in the patients with COPD $(1,480.7 \pm 256.7)$, compared with the non-COPD subjects $(1,954.1 \pm 312.2$; Fig. 1B).

SP-A-positive type II pneumocytes. The alveolar macrophages and certain type II pneumocytes were SP-A-positive. The expression of TTF-1 was only detected in the type II pneumocytes, with the alveolar macrophages being TTF-1-negative. As type II pneumocytes and alveolar macrophages have morphological differences, immunostaining with TTF-1 and SP-A monoclonal antibodies was used to determine the total 
A

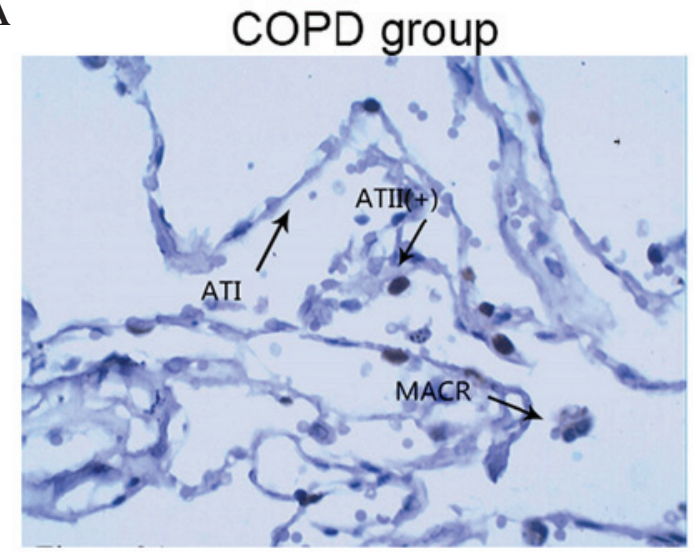

B

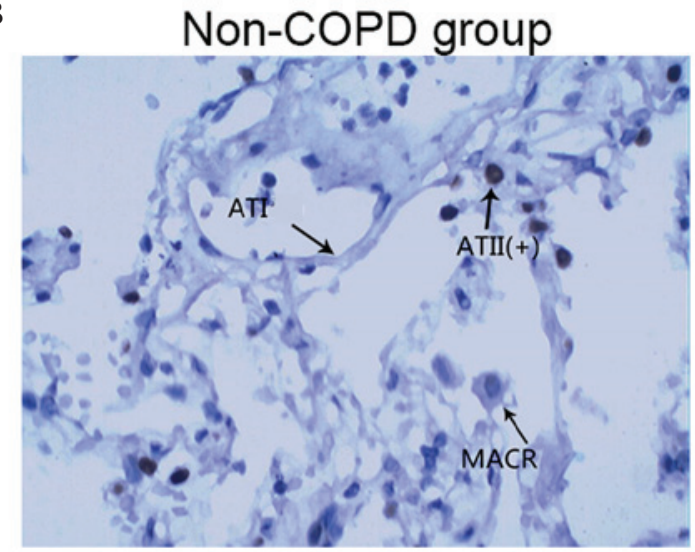

Figure 3. Immunostaining of TTF-1 in human lung tissue (magnification, x400). Representative TTF-1-immunostained images from (A) a patient with COPD and (B) a subject without COPD. ATII (+) indicates SP-A-positive type II pneumocytes; ATII (-) indicates SP-A-negative type II pneumocytes; MACR represents alveolar macrophages. COPD, chronic obstructive pulmonary disease; TFF-1, thyroid transcription factor-1.

A

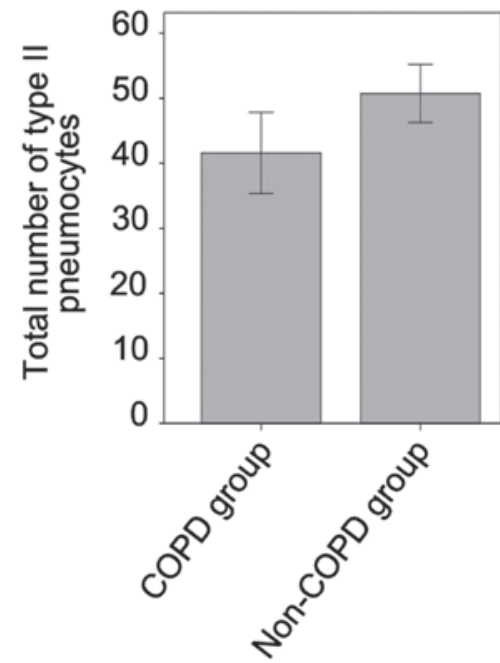

B

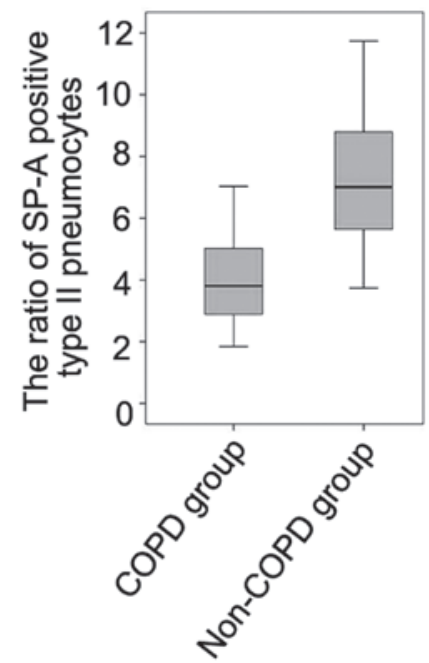

Figure 4. Type II pneumocytes. (A) Total number of type II pneumocytes. (B) Ratio of SP-A-positive type II pneumocytes (\%). Data are expressed as the mean \pm standard deviation. COPD, chronic obstructive pulmonary disease; SP-A, surfactant protein A.

A

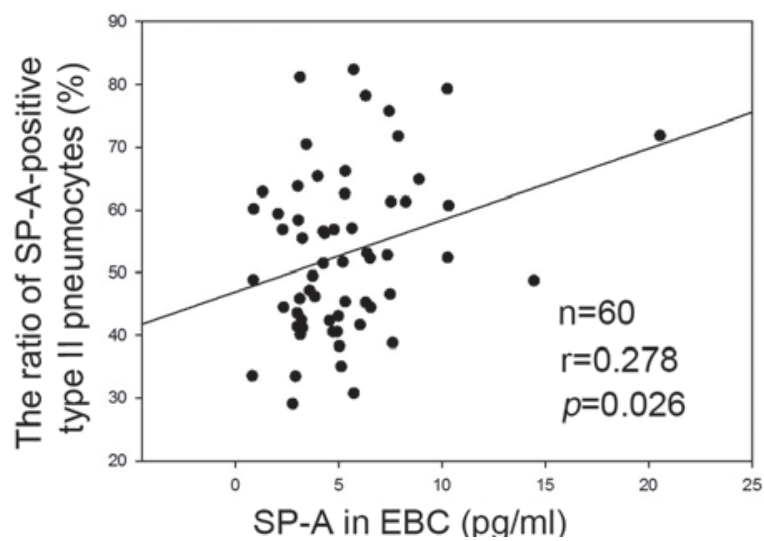

B

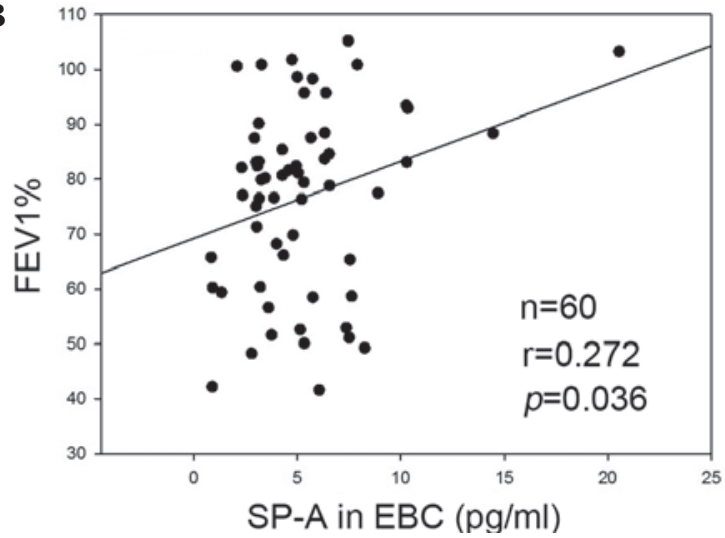

Figure 5. Correlation between the expression levels of P-A and EBC. (A) Expression levels of SP-A in the EBC were correlated with the ratio of SP-A-positive type II pneumocytes/total type II pneumocytes ( $\mathrm{r}=0.278 ; \mathrm{P}=0.026$ ). (B) Expression levels of SP-A in the EBC were correlated with the predicted FEV1\% $(\mathrm{r}=0.272 ; \mathrm{P}=0.036)$. EBC, exhaled breath condensate; SP-A, surfactant protein A; FEV1, forced expiratory volume in one second.

number of type II pneumocytes and SP-A-positive type II pneumocytes. Images of the SP-A immunostained tissues from a patient with COPD and a patient without COPD are shown in Fig. 2A and B, respectively. The images in Fig. 3 show 
representative TTF-1-immunostained images of lung tissue samples from a patient with COPD (Fig. 3A) and a patient without COPD (Fig. 3B). Although no significat difference was detected, the results demonstrated that the total number of type II pneumocytes was lower in the COPD group, compared with the non-COPD group, and the ratio of SP-A-positive type II pneumocytes (SP-A-positive type II pneumocytes/total type II pneumocytes, expressed as a percentage) was decreased in the COPD group, compared with the non-COPD group (Figs. 2-4).

Correlation between the expression levels of $S P-A$ and $E B C$. As shown in Fig. 5A, the expression levels of SP-A in the EBC were correlated with the ratio of SP-A-positive type II pneumocytes/total type II pneumocytes $(\mathrm{r}=0.278 ; \mathrm{P}=0.026)$. Decreased expression levels of SP-A were associated with a decreased ratio of SP-A-positive type II pneumocytes. In addition, the ratio of SP-A-positive type II pneumocytes was correlated with the predicted FEV $1 \%$ (data not shown), suggesting that the increased degree of airway obstruction was associated with an increase in the SP-A ratio. The expression levels of SP-A in the EBC were also correlated with the predicted FEV 1\% ( $\mathrm{r}=0.272 ; \mathrm{P}=0.036)$, suggesting that the decreased expression levels of SP-A in EBC were associated with an increase in the degree of airway obstruction (Fig. 5B).

\section{Discussion}

The non-invasive measurement of airway obstruction in patients with COPD may prove useful for monitoring the disease. EBC analysis is a non-invasive method for detecting airway lining fluid composition, and the technique has numerous advantages, as it is easy to perform and less expensive in terms of equipment and personnel costs, compared with sampling BAL and induced sputum $(25,26)$.

Controversial results have been reported regarding the expression levels of SP-A in the BAL or serum of patients with COPD. Decreased expression levels of SP-A have been reported in smokers who are otherwise healthy, elderly people and patients with COPD $(19,20,27)$. Ohlmeier et al (28) reported increased expression levels of SP-A in lung tissue samples and the induced sputum of patients with COPD, compared with normal or fibrotic lung tissue samples. Vlachaki et al (21) also reported that decreased expression levels of SP-A in lung tissue samples of patients with COPD are associated with the pathogenesis of COPD.

In the present study, investigation to identify a non-invasive SP-A measurement technique and subsequent investigation of the expression levels of SP-A in patients with COPD were performed. The expression levels of SP-A were determined in EBC collected, which was collected from patients with COPD and non-COPD subjects. The results of the present study demonstrated that the expression levels of SP-A were significantly decreased in the patients with COPD, compared with the non-COPD subjects, and the expression of SP-A in the EBC was correlated with that in the lung tissue. Immunostaining of TTF-1 revealed that the total number of type II pneumocytes in patients with COPD was increased, compared with the number in the non-COPD subjects. The decrease in the total number of type II pneumocytes may be due to the extensive tissue damage in COPD (12), and has been reported in previous studies following lung injury $(29,30)$. Type II pneumocytes proliferate during lung injury and chronic inflammatory states, including COPD, in order to produce type I neumocytes and specific products, including SP-A), which are involved in lung defense (31).

The results of the present study demonstrated that the ratio of SP-A-positive type II pneumocytes to the overall type II pneumocytes was decreased in patients with COPD, compared with the non-COPD subjects. One possible explanation is that disease-induced epithelial destruction depletes the type II pneumocytes, which are responsible for the production of SP-A. Furthermore, the decreased expression levels of SP-A in EBC were associated with a higher degree of airway obstruction, suggesting that the measurement of SP-A in EBC may be a potential method for monitoring airway obstruction in patients with COPD.

In conclusion, the present study performed non-invasive SP-A measurement by detecting the expression levels of SP-A in EBC collected from patients with COPD. Decreased expression levels of SP-A in the EBC were observed in the patients with COPD, compared with the non-COPD subjects, and these decreased expression levels were also observed in the lung tissues. In addition, the total number of type II pneumocytes in the patients with COPD were decreased and the ratios of SP-A-positive type II pneumocytes were reduced, compared with those in the non-COPD subjects. The reduction in SP-A-positive type II pneumocytes was associated with the expression levels of SP-A. Finally, the results of the present study demonstrated that decreased expression levels of SP-A in the EBC were associated with a higher degree of airway obstruction. These results suggested that the measurement of SP-A in EBC may be a potential method for monitoring airway obstruction in patients with COPD. Further investigations are required in order to further examine these observations and elucidate the underlying mechanisms.

\section{Acknowledgements}

This present study was funded by grants from the Tianjin Municipal Science and Technology Commission (grant no. 13ZCZDSY01800) and the Tianjin Health Bureau of Science and Technology Funds (grant no. 2012KR16).

\section{References}

1. Vestbo J, Hurd SS, Agusti AG, Jones PW, Vogelmeier C, Anzueto A, Barnes PJ, Fabbri LM, Martinez FJ, Nishimura M, et al: Global strategy for the diagnosis, management and prevention of chronic obstructive pulmonary disease: GOLD executive summary. Am J Respir Crit Care Med 187: 347-365, 2013.

2. Barnes PJ: Chronic obstructive pulmonary disease. N Engl J Med 343: 269-280, 2000.

3. Pellegrino R and Brusasco V: On the causes of lung hyperinflation during bronchoconstriction. Eur Respir J 10: 468-475, 1997.

4. Baldi S, Miniati M, Bellina CR, Battolla L, Catapano G, Begliomini E, Giustini D and Giuntini C: Relationship between extent of pulmonary emphysema by high-resolution computed tomography and lung elastic recoil in patients with chronic obstructive pulmonary disease. Am J Respir Crit Care Med 164: 585-589, 2001 
5. Rutgers SR, Timens W, Kaufmann HF, van der Mark TW, Koëter GH and Postma DS: Comparison of induced sputum with bronchial wash, bronchoalveolar lavage and bronchial biopsies in COPD. Eur Respir J 15: 109-115, 2000.

6. Bhowmik A, Seemungal TA, Sapsford RJ, Devalia JL and Wedzicha JA: Comparison of spontaneous and induced sputum for investigation of airway inflammation in chronic obstructive pulmonary disease. Thorax 53: 953-956, 1998.

7. Saetta M, Turato G, Maestrelli P, Mapp CE and Fabbri LM Cellular and structural bases of chronic obstructive pulmonary disease. Am J Respir Crit Care Med 163: 1304-1309, 2001.

8. Wielders PL and Dekhuijzen PN: Disease monitoring in chronic obstructive pulmonary disease: Is there a role for biomarkers? Eur Respir J 10: 2443-2445, 1997.

9. Hunt J: Exhaled breath condensate: An evolving tool for noninvasive evaluation of lung disease. J Allergy Clin Immunol 110 28-34, 2002

10. Carter SR, Davis CS and Kovacs EJ: Exhaled breath condensate collection in the mechanically ventilated patient. Respir Med 106: 601-613, 2012

11. Anzueto A, Jubran A, Ohar JA, Piquette CA, Rennard SI, Colice G, Pattishall EN, Barrett J, Engle M, Perret KA and Rubin BK: Effects of aerosolized surfactant in patients with stable chronic bronchitis: A prospective randomized controlled trial. JAMA 278: 1426-1431, 1997.

12. Bernhard W, Haslam PL and Floros J: From birds to humans: New concepts on airways relative to alveolar surfactant. Am J Respir Cell Mol Biol 30: 6-11, 2004.

13. Milic-Emili J: Does mechanical injury of the peripheral airways play a role in the genesis of COPD in smokers? COPD 1: 85-92, 2004.

14. Sin DD, Pahlavan PS and Man SF: Surfactant protein D: A lung specific biomarker in COPD? Ther Adv Respir Dis 2: 65-74, 2008

15. Guo X, Lin HM, Lin Z, Montaño M, Sansores R, Wang G, DiAngelo S, Pardo A, Selman M and Floros J: Polymorphisms of surfactant protein gene A, B, D, and of SP-B-linked microsatellite markers in COPD of a Mexican population. Chest 117: 249S-250S, 2000

16. Otto-Verberne CJ, Ten Have-Opbroek AA, Franken C, Hermans J and Dijkman JH: Protective effect of pulmonary surfactant on elastase-induced emphysema in mice. Eur Respir J 5: 1223-1230, 1992.

17. Kishore U, Greenhough TJ, Waters P, Shrive AK, Ghai R, Kamran MF, Bernal AL, Reid KB, Madan T and Chakraborty T: Surfactant proteins SP-A and SP-D: Structure, function and receptors. Mol Immunol 43: 1293-1315, 2006.
18. Pastva AM, Wright JR and Williams KL: Immunomodulatory roles of surfactant proteins A and D: Implications in lung disease. Proc Am Thorac Soc 4: 252-257, 2007.

19. Honda Y, Takahashi H, Kuroki Y, Akino T and Abe S: Decreased contents of surfactant proteins A and D in BAL fluids of healthy smokers. Chest 109: 1006-1009, 1996.

20. Betsuyaku T, Kuroki Y, Nagai K, Nasuhara Y and Nishimura M: Effects of ageing and smoking on SP-A and SP-D levels in bronchoalveolar lavage fluid. Eur Respir J 24: 964-970, 2004.

21. Vlachaki EM, Koutsopoulos AV, Tzanakis N, Neofytou E, Siganaki M, Drositis I, Moniakis A, Schiza S, Siafakas NM and Tzortzaki EG: Altered surfactant protein-A expression in type II pneumocytes in COPD. Chest 137: 37-45, 2010.

22. Rahman I and Biswas SK: Non-invasive biomarkers of oxidative stress: Reproducibility and methodological issues. Redox Rep 9: 125-143, 2004.

23. Celli BR and MacNee W; ATS/ERS Task Force: Standards for the diagnosis and treatment of patients with COPD: A summary of the ATS/ERS position paper. Eur Respir J 23: 932-946, 2004.

24. Mutti A, Corradi M, Goldoni M, Vettori MV, Bernard A and Apostoli P: Exhaled metallic elements and serum pneumoproteins in asymptomatic smokers and patients with COPD or asthma. Chest 129: 1288-1297, 2006.

25. Kharitonov SA and Barnes PJ: Exhaled markers of pulmonary disease. Am J Respir Crit Care Med 163: 1693-1722, 2001.

26. Jöbsis Q, Raatgeep HC, Schellekens SL, Kroesbergen A, Hop WC and de Jongste JC: Hydrogen peroxide and nitric oxide in exhaled air of children with cystic fibrosis during antibiotic treatment. Eur Respir J 16: 95-100, 2000

27. Guo X, Lin HM, Lin Z, Montaño M, Sansores R, Wang G, DiAngelo S, Pardo A, Selman M and Floros J: Surfactant protein gene A, B and D marker alleles in chronic obstructive pulmonary disease of a Mexican population. Eur Respir J 18: 482-490, 2001.

28. Ohlmeier S, Vuolanto M, Toljamo T, Vuopala K, Salmenkivi K, Myllärniemi M and Kinnula VL: Proteomics of human lung tissue identifies surfactant protein A as a marker of chronic obstructive pulmonary disease. J Proteome Res 7: 5125-5132, 2008.

29. Wirtz HR and Schmidt M: Acute influence of cigarette smoke on secretion of pulmonary surfactant in rat alveolar type II cells in culture. Eur Respir J 9: 24-32, 1996.

30. Subramaniam S, Whitsett JA, Hull W and Gairola CG: Alteration of pulmonary surfactant proteins in rats chronically exposed to cigarette smoke. Toxicol Appl Pharmacol 140: 274-280, 1996.

31. Garcia O, Hiatt MJ, Lundin A, Lee J, Reddy R, Navarro S, Kikuchi A and Driscoll B: Targeted type 2 alveolar cell depletion provides a dynamic functional model for lung injury repair. Am J Respir Cell Mol Biol:Jul 23, 2015 (Epub ahead of print). 